\title{
Malignant Middle Cerebral Artery Infarction Secondary to Traumatic Bilateral Internal Carotid Artery Dissection. A Case Report
}

\author{
Zoltán Bajkó1, Rodica Bălaşa ${ }^{1}$, Anca Moţăţăianu ${ }^{1 *}$, Laura Bărcuţean ${ }^{1}$, Adina Stoian², \\ Nicoleta Stirbu ${ }^{3}$, Smaranda Maier ${ }^{1}$ \\ ${ }^{1}$ University of Medicine and Pharmacy Târgu Mureş, Department of Neurology \\ 2 University of Medicine and Pharmacy Târgu Mureş, Department of Pathophysiology \\ ${ }^{3}$ Mediab Medical Center Târgu Mureş
}

\begin{abstract}
Traumatic bilateral dissection of the carotid arteries is a rare condition with potentially life-threatening complications. The case of a 57-year-old male patient with acute onset left sided hemiparesis, twelve hours after a blunt head injury, caused by a horse kick, is reported. A cerebral CT scan revealed right middle cerebral artery (MCA) territory infarction. Based on Duplex ultrasound and Angio CT scan findings, a diagnosis of bilateral ICA dissection was established. Despite antithrombotic treatment, the patient presented with a progressive worsening of his neurological status. The control CT scan evidenced malignant right MCA territory infarction that required decompressive craniotomy. The patient was discharged with significant neurological deficits. Together with this case, the aetiologies, clinical manifestations, diagnostic and therapeutical options and outcome of carotid artery dissection are discussed.
\end{abstract}

Keywords: carotid artery dissection, malignant infarction, traumatic

Received: 26 April 2016 / Accepted: 30 May 2016

\section{INTRODUCTION}

Bilateral traumatic dissection of the carotid arteries is a rare and potentially life-threatening condition. It can often be overlooked, due to the unremarkable associated trauma and the delay in onset of any neurological events $[1,2]$.

\section{CASE REPORT}

This is a report concerning a patient with blunt head injury secondary to a horse kick, who presented twelve hours after the trauma with progressive neurological signs suggestive of right middle cerebral artery (MCA) stroke, developing malignant MCA infarction, which was treated by decompressive craniotomy.

A 57-year-old male patient who was admitted to the Neurology Department with left-sided hemiparesis, outside the time window for thrombolysis. His medical history was unremarkable, with the exception of a history of chronic heavy alcohol consumption. Twelve hours before the onset of left sided weakness he had suffered a blunt head injury, from a horse kick.

A physical examination revealed a minimal left frontal post-traumatic injury. Neurologic examination indicated left central facial palsy, left hemiplegia, diminished left deep tendon reflexes, left extensor plantar response and left superficial hemi hypoesthesia, with a score of 16 on NIH stroke scale.

The laboratory results and ECG were normal.

A cranial CT scan revealed a right MCA infarction, without significant mass effect and a hyper dense MCA sign (Figure 1).

A Duplex ultrasound examination of the cervical vessels revealed a high-resistance flow signal in the right

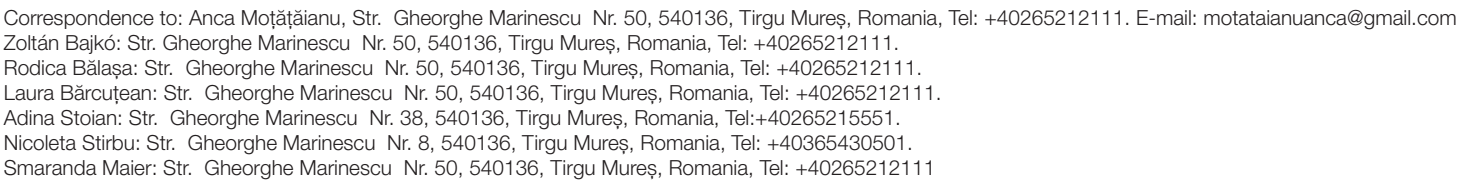




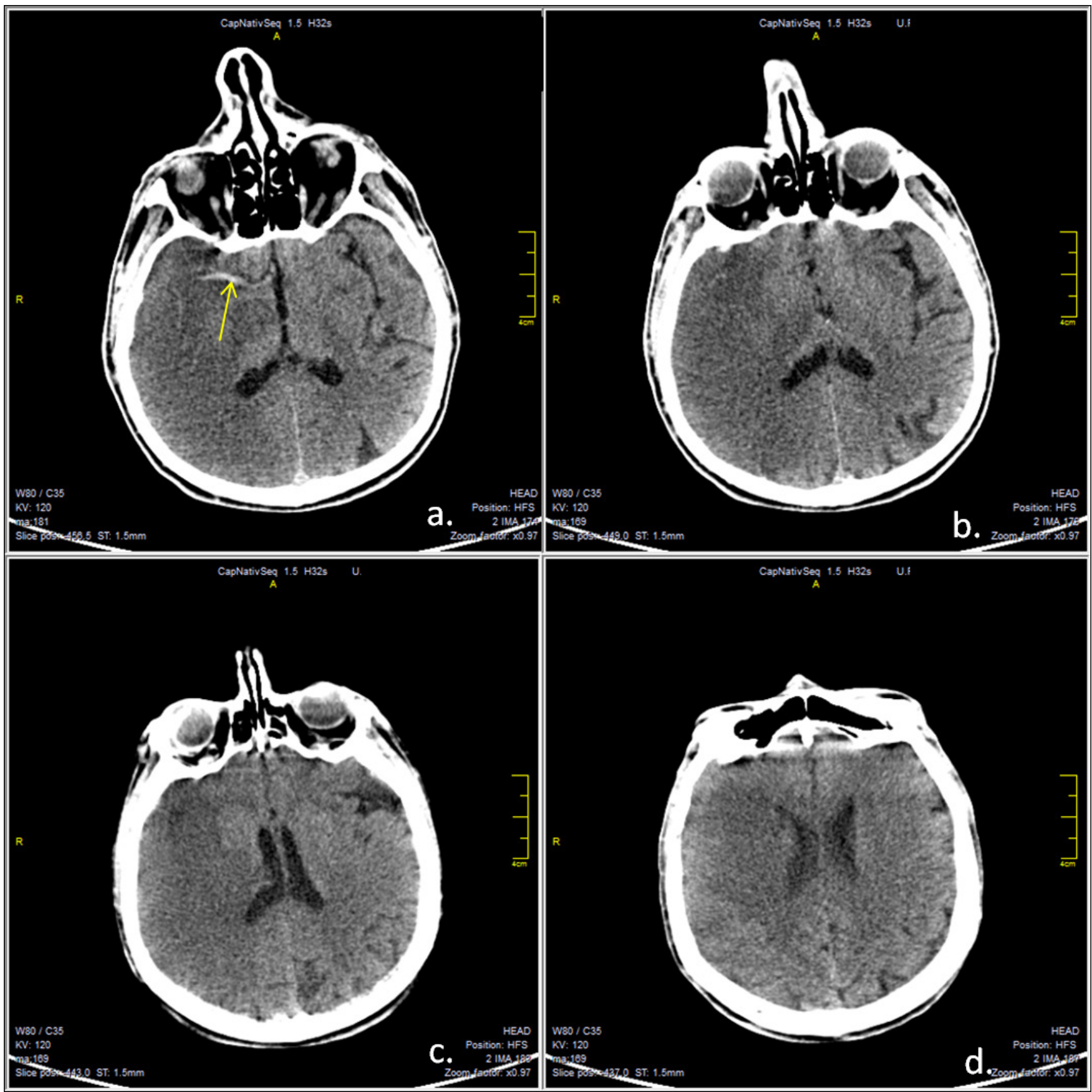

Fig. 1. Native cerebral CT scan, axial sections revealing right middle cerebral territory infarction, without significant midline shift. Hyperdense middle cerebral artery sign is visible (a, yellow arrow).

internal carotid artery (ICA), without stenotic lesions at the proximal level, suggestive of a significant distal stenosis or occlusion. (Figure 2a, 2b) Ultrasound examination of the left ICA revealed irregular stenosis caused by a hypoechoic mural thickening, suggestive of a mural hematoma secondary to dissection (Figure $2 \mathrm{c}, 2 \mathrm{~d}$ ).

A CT angiography examination of the cervical vessels showed along-segment, high-grade stenosis (string sign) of the distal segment, indicative of dissection at the level of the right ICA (Figure 3).
Because the large MCA infarction was considered to be associated with a high risk of haemorrhage, anticoagulation treatment was postponed. The patient was treated with antiplatelets (Aspirin) and low molecular weight heparin in thrombo-prophylactic doses.

Three days after the admission, the patient presented with progressive alteration of consciousness with a Glasgow coma score of 10 . The control cranial CT scan indicated a right MCA infarction with substantial mass effect against the midline structures, which were shifted 


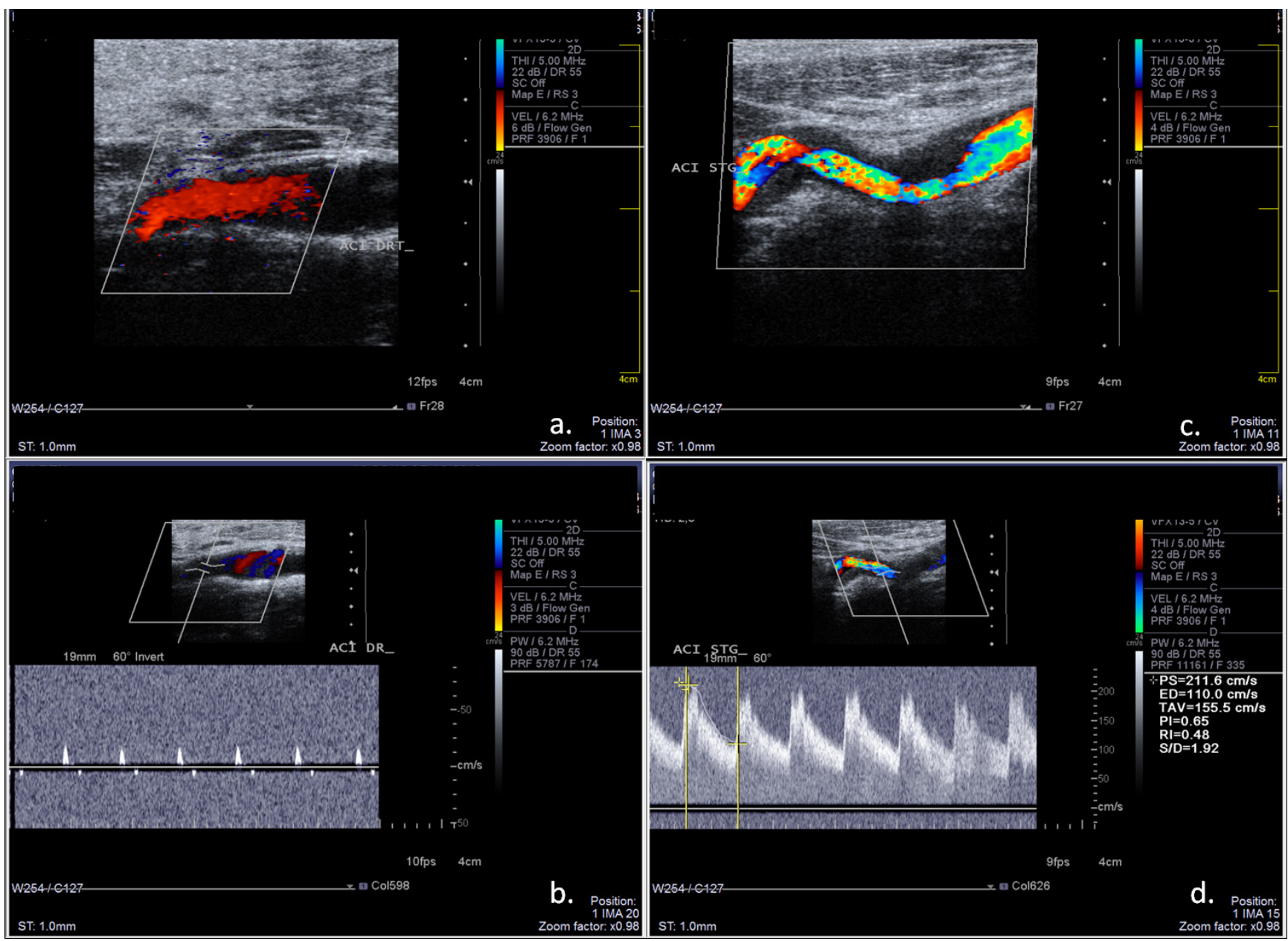

Fig. 2. Ultrasound examination of the carotid arteries. a: color mode examination of the right ICA, longitudinal section, without stenotic lesions; b: real-time triplex display (Color Doppler+ Pulsed Spectral Doppler) of the right ICA revealing high-resistance flow signal, with low and short systolic flow and completely absent diastolic flow, indicative of near occlusion or occlusion of the distal segment of ICA; c: color-mode examination of the left ICA revealing irregular stenosis caused by the hypoechoic mural hematoma; $d$ : triplex mod examination of the left ICA, revealing high flow velocities suggestive of significant stenosis.

$12 \mathrm{~mm}$ to the left (Figure 4). The patient was transferred to the neurosurgical department of the hospital for emergency decompressive craniotomy, on the advice of the duty neurosurgeon. The control CT scan, after decompression, showed a mild reduction of the mass effect, with the left shift reduced to $8 \mathrm{~mm}$ (Figure 5).

Following the decompression surgery, the neurological status of the patient stabilized and progressively the patient regained consciousness.

Oral anticoagulation therapy was initiated three weeks after the ischemic event, and the neurological status of the patient showed a mild improvement Grade 1 motor deficit at the upper extremity, and a Grade 2 deficit at lower extremity was recorded before discharge from the neurosurgical department. The patient was transferred to a rehabilitation ward.

\section{Discussion}

The dissection of the cervical arteries is responsible for approximately $2 \%$ of all ischaemic cerebrovascular events and up to $25 \%$ of stroke in young adult patients. It occurs predominantly in the middle adult years with males being more frequently affected [3].

The majority of dissections result from minor or trivial injury or occur spontaneously. Only rarely do cases results from major head or neck trauma. Trauma can be direct, such as blunt trauma, penetrating injury, iatrogenic-endovascular intervention, or indirect, such as sudden movement causing hyperextension of the neck [4].

Dissections usually affect one cervical vessel, with the extracranial segment of the arteries being most fre- 


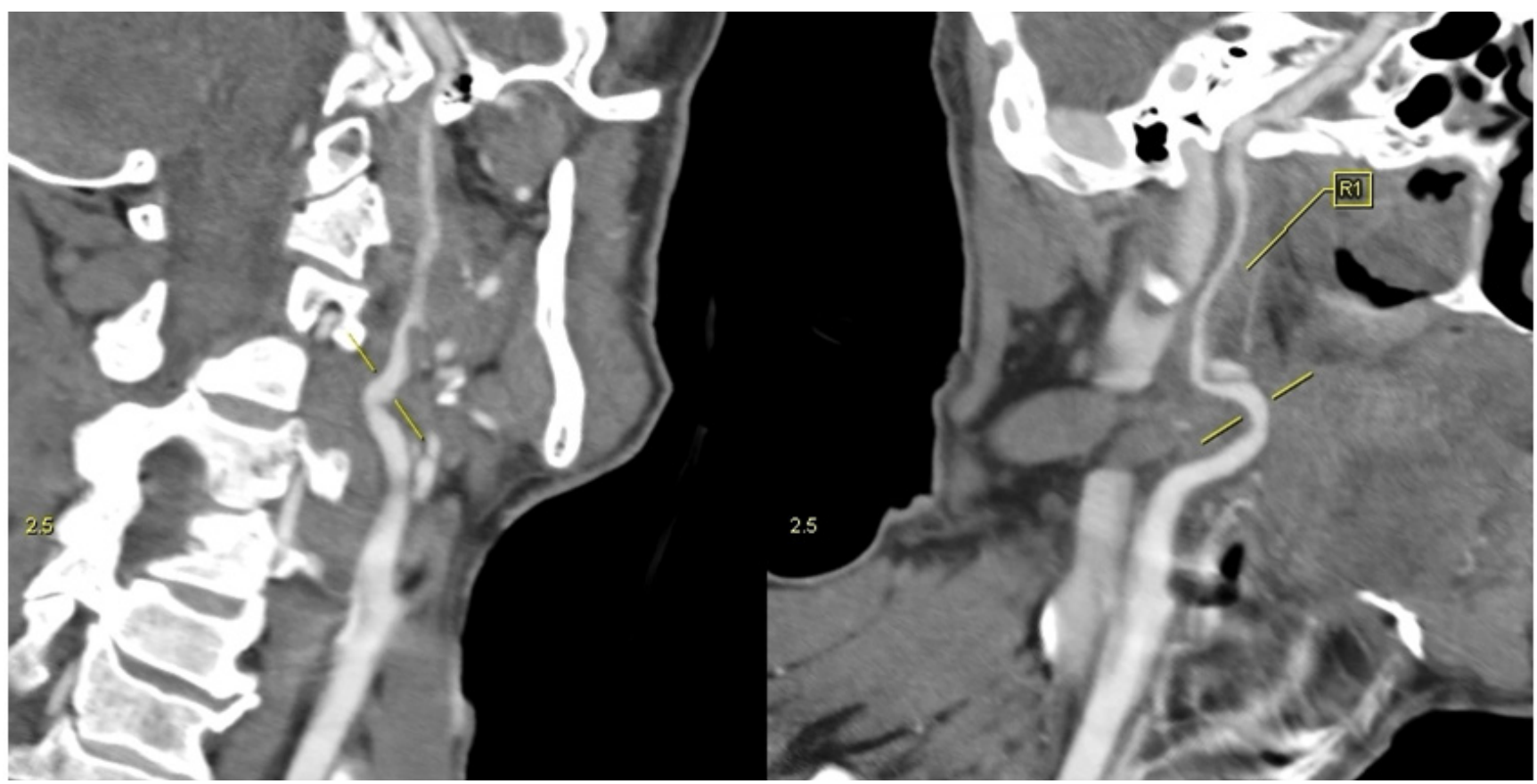

Fig. 3. Angio CT scan examination of the right ICA, curved planar reformatted images, revealing long-segment highgrade stenosis (string sign) of the distal segment of the vessel, indicative of dissection.

quently affected. Multiple simultaneous dissections are found in 13-22\% of the cases [5-7].

Bilateral traumatic ICA dissection is rare, with only a few cases reported in the literature [1,2]. Traumatic dissection usually affects the mobile, cervical segment of the carotid and vertebral arteries. Most commonly involved is the pre-petrous segment of the ICA, between the carotid bulb and the entry into the petrous temporal bone. At this level, the ICA is compressed against the transverse processes of the vertebra and $\mathrm{C} 1-\mathrm{C} 2$ nerves, and a mural hematoma forms on the posterior wall.

Carotid dissections have been associated with an elongated styloid process and tortuosity, kinking, coiling or loops of the carotid vessel [8].

Many patients with dissection have concomitant cardiovascular risk factors such as atherosclerosis, arterial hypertension, migraine, and excessive alcohol consumption. Hypertension seems to be a major risk factor for dissection. Genetic predisposition in cases such Ehlers-Danlos syndrome and Marfan syndrome, with fibromuscular dysplasia and cystic media necrosis, is well documented [9].

The clinical manifestations of a dissection include ischaemic stroke or transient ischemic attack, subarachnoid haemorrhage, and local symptoms as neck pain and headache. Asymptomatic cases must not be underestimated [4].
Approximately $50 \%$ of cases of dissection develop stroke and $30 \%$ present with TIA. Occlusive lesions lead to larger infarcts, with most of the infarcts occurring within the first 3-5 days after the dissection [10].

Comparing the characteristics of carotid and vertebral artery dissections, those in which the carotid is affected were older, more often male, had a higher frequency of infection, lower frequency of trauma, had more severe stroke and worse prognosis, increased incidence of persistent neurological insufficiencies, despite the fact that their rate of stroke was lower than those with vertebral artery dissections $[9,11]$.

The mechanism of cerebral ischemia in dissections is mainly artery-to-artery embolism. Hemodynamic compromise is rarely recognized as the primary mechanism of ischemia in dissections [12].

The diagnosis of dissection is based on the neuro-imaging findings. The main features suggestive of dissection are a mural hematoma, the long-segment tapering stenosis (string sign), tapering occlusion (flame-shaped occlusion), pseudo aneurysm, intimal flap, and double lumen. Extracranial carotid dissections, typically occur $2 \mathrm{~cm}$ or more distal to the bifurcation of the carotid artery, at the base of the skull.

Carotid Duplex ultrasonography and a transcranial Doppler ultrasound test is useful for screening suspected carotid dissection, or monitoring the recanali- 

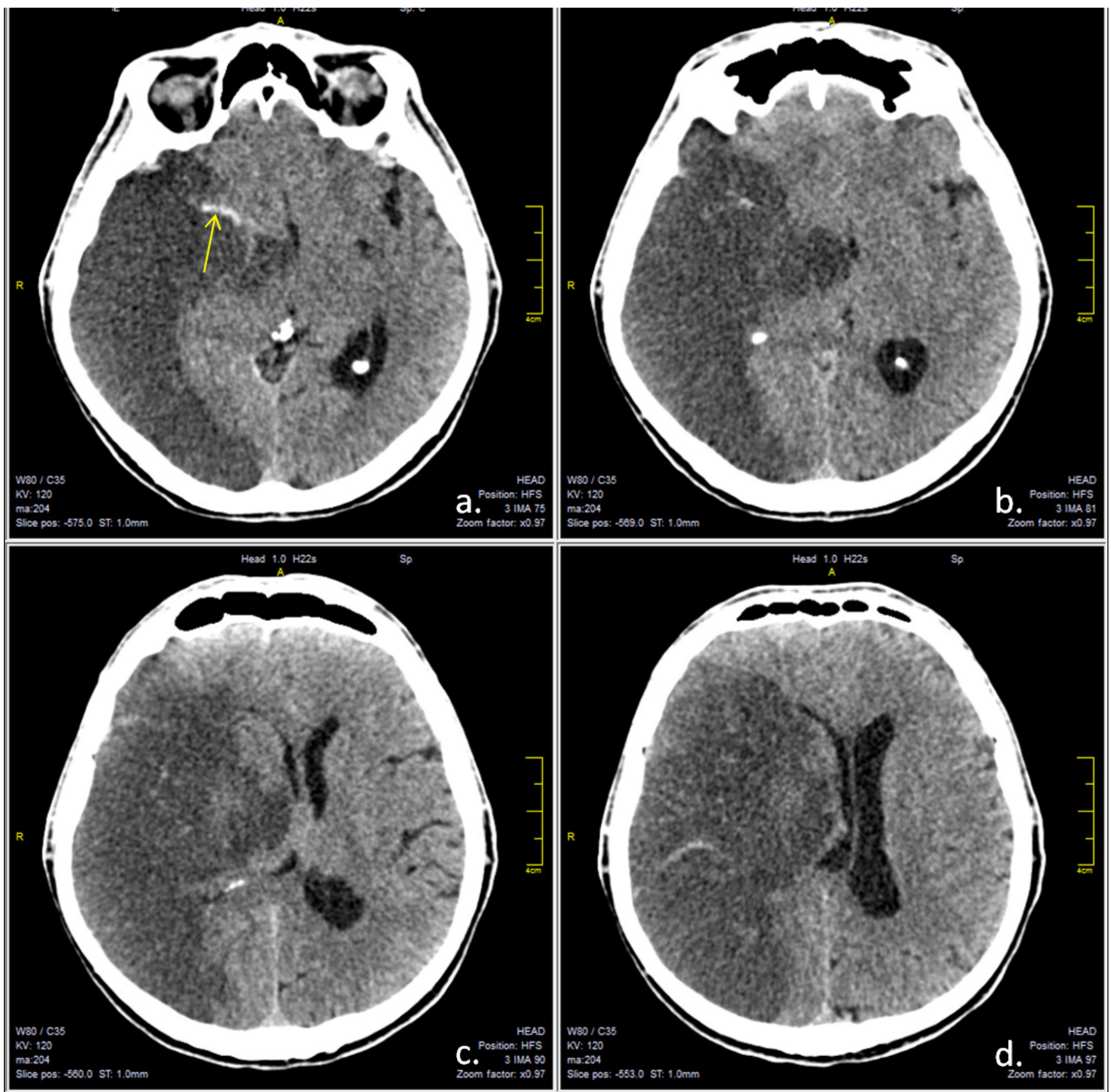

Fig. 4. Native cerebral CT scan, axial sections revealing right middle cerebral territory infarction, with important mass effect and significant midline shift. Hyperdense middle cerebral artery sign is visible (a, yellow arrow).

zation process. The main limitations of this method are that it cannot visualize the lesions near the skull base, is operator dependent, and usually cannot detect pseudo aneurysms.

In the present case, ultrasound examination of the right ICA (symptomatic side) only revealed an indirect sign of significant distal pathology, but based on this finding and the medical history of blunt trauma, an angio-CT scan was considered justified. On the left ICA (asymptomatic side) direct ultrasound findings of irregular stenosis and a hypoechoic mural hematoma were very suggestive of the occurrence of dissection.

MRI combined with MR angiography is most frequently used in establishing a diagnosis of dissection, as they can visualize the pathognomonic crescent sign of an intramural hematoma, which is usually described in T1-weighted fat-saturation sequences, and is described as an eccentric rim of hyper intensity surrounding the hypo intense arterial lumen. 

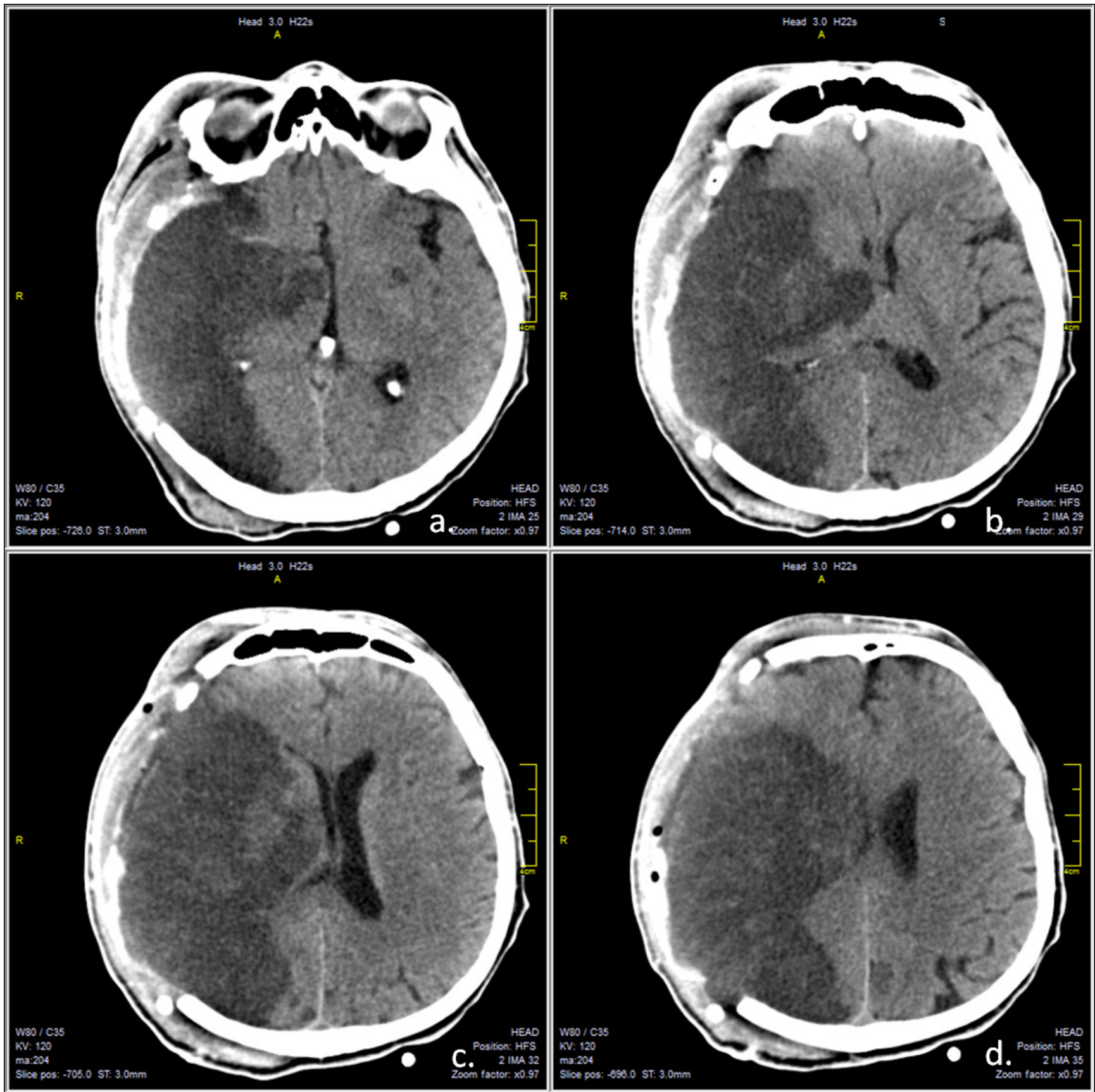

Fig. 5. Native cerebral CT scan, axial sections after decompressive craniectomy, revealing right middle cerebral territory infarction, with slightly diminished midline shift.

The sensitivity and specificity of CTA for the diagnosis of dissections are similar to MR techniques.

Conventional angiography is the gold standard for diagnosis and is used when the clinical suspicion of dissection remains high despite negative non-invasive diagnostic results [4].

In a case of dissection associated with cerebral infarction, the treatment options are similar to the standard treatment of ischaemic stroke associated with other aetiologies. Thrombolysis must be considered in eligible patients. There is a controversy regarding antithrombotic treatment. The efficiency of antiplatelet and anticoagulant treatment both seems to be similar. However in the case of extracranial dissection associated with stroke, anticoagulant treatment may be a better option. In the case of intracranial dissection and dissection without stroke, antiplatelet treatment appears to be more suitable. Endovascular treatment is mainly used in cases with recurrent ischemia despite conservative treatment [4]. 
The prognosis of dissections depends on the severity of the secondary ischaemic stroke. In patients with extracranial dissection, excellent recovery occurs in 70$85 \%$ of cases, severe neurological deficits in $10-25 \%$, and death occurs in 5-10\% [4].

Malignant MCA infarction is a well-recognized entity and represents $10-15 \%$ of cerebral infarctions. Because both severe cerebral oedema and increased intracranial pressure lead to trans-tentorial and uncal herniation in $80 \%$ of the cases, the mortality of this lifethreatening condition is also about $80 \%$.

Decompressive hemi-craniotomy is a possible lifesaving option which can decrease the mortality rates from 80 to $30 \%[13,14]$.

\section{CONCLUSIONS}

Carotid artery dissection is an important cause of stroke in young patients. Physicians must be aware of this potentially life threatening condition, and targeted neuro-imaging tests should be performed for a proper diagnosis. Neurological symptoms can be delayed following trauma. In cases of blunt cervical trauma, especially if the patient presents with local symptoms such as head and neck pain, Horner syndrome, tinnitus, local bruit, or cranial neuropathies, screening for a carotid artery dissection must be undertaken even when signs of ischemic stroke are not present.

The diagnosis and treatment are sometimes challenging but early diagnosis and proper therapy result in favourable outcomes.

\section{CONFLICT OF INTEREST}

None declared

\section{REFERENCES}

1. Crönlein M, Sandmann GH, Beirer M, Wunderlich S, Biberthaler $P$, Huber-Wagner S. Traumatic bilateral carotid artery dissection following severe blunt trauma: a case report on the difficulties in diagnosis and therapy of an often overlooked life-threatening injury. Eur J Med Res. 2015;20:62.

2. Samra NS, Ravi AK, Johnson LW, Williams M. Traumatic bilateral carotid artery dissection. J La State Med Soc. 2011;163:26-8.

3. Arnold M, Kappeler L, Georgiadis D, et al. Gender differences in spontaneous cervical artery dissection. Neurology. 2006;67:1050-2.

4. Liebeskind DS, Saver J. Spontaneous cerebral and cervical artery dissection: clinical features and diagnosis. UpToDate. 2016; cerebrovascular diseases, http://www.uptodate.com/contents/ spontaneous-cerebral-and-cervical-artery-dissection-clinicalfeatures-and-diagnosis

5. Chaves C, Estol C, Esnaola MM, et al. Spontaneous intracranial internal carotid artery dissection: report of 10 patients. Arch Neurol. 2002;59:977-81.

6. Lee VH, Brown RD Jr, Mandrekar JN, Mokri B. Incidence and outcome of cervical artery dissection: a population-based study. Neurology. 2006;67:1809-12.

7. Hassan $\mathrm{AE}$, Zacharatos $\mathrm{H}$, Mohammad $\mathrm{YM}$, et al. Comparison of single versus multiple spontaneous extra- and/or intracranial arterial dissection. J Stroke Cerebrovasc Dis. 2013;22:42-8.

8. Singhal AB. Spontaneous carotid and vertebral artery dissection. In: Levine SR, editor. MedLink Neurology. San Diego: MedLink Corporation, 2013

9. Debette S, Metso T, Pezzini A, et al. Association of vascular risk factors with cervical artery dissection and ischemic stroke in young adults. Circulation. 2011;123:1537-44.

10. Naggara O, Morel A, Touzé E, et al. Stroke occurrence and patterns are not influenced by the degree of stenosis in cervical arterydissection. Stroke. 2012;43:1150-2.

11. Chandra A, Suliman A, Angle N. Spontaneous dissection of the carotid and vertebral arteries: the 10-year UCSD experience. Ann Vasc Surg. 2007;21:178-85.

12. Lucas C, Moulin T, Deplanque D, Tatu L, Chavot D. Stroke patterns of internal carotid artery dissection in 40 patients. Stroke. 1998;29:2646-8.

13. Rahmanian A, Seifzadeh B, Razmkon A, et al. Outcome of decompressive craniectomy in comparison to nonsurgical treatment in patients with malignant MCA infarction. Springerplus. 2014;3:115.

14. Staykov D, Gupta R. Hemicraniectomy in malignant middle cerebral artery infarction. Stroke. 2011;42:513-6. 